\title{
Computational Problems in $S$-Function Theory
}

\author{
By P. A. Morris
}

\begin{abstract}
In this paper we discuss some computational problems associated with Schurfunctions. A well-known algorithm for the ordinary product is described and adapted for a computer. A theorem of Todd is discussed in the same way, and these methods are combined to produce a general program for the plethysm (wreath product) of two $S$-functions.
\end{abstract}

1. Introduction. In the theory of the symmetric group and of its representations, Schur-functions, or $S$-functions, are defined either in terms of the homogeneous product-sum symmetric functions $h_{r}$ or in terms of the characters of the symmetric group ([3], [4]). Given a number $n$, to each partition $(\lambda) \equiv\left(\lambda_{1}, \lambda_{2}, \cdots, \lambda_{n}\right)$ of $n$ there corresponds an $S$-function $\{\lambda\} \equiv\left\{\lambda_{1}, \lambda_{2}, \cdots, \lambda_{n}\right\}$ defined, in terms of the functions $h_{r}$, by

$$
\{\lambda\} \equiv\left\{\lambda_{1}, \lambda_{2}, \cdots, \lambda_{n}\right\}=\left|h_{\lambda_{s-s+l}}\right| .
$$

For example, the $S$-function

$$
\{3,2,1\}=\left|\begin{array}{lll}
h_{3} & h_{4} & h_{5} \\
h_{1} & h_{2} & h_{3} \\
h_{-1} & h_{0} & h_{1}
\end{array}\right|=\left|\begin{array}{ccc}
h_{3} & h_{4} & h_{5} \\
h_{1} & h_{2} & h_{3} \\
0 & 1 & h_{1}
\end{array}\right|
$$

(since $h_{0}=1, h_{r}=0$ when $r<0$ ).

We shall always write the $S$-functions with $\lambda_{1} \geqq \lambda_{2} \geqq \cdots \geqq \lambda_{n} \geqq 0$. In that case we call the number of nonzero $\lambda$ 's the length of the $S$-function. If the length is one, we say that the $S$-function is one-part and we have

$$
\left\{\lambda_{1}\right\}=\left|h_{\lambda_{1}}\right|=h_{\lambda_{1}} \text {. }
$$

Thus, for example $\{4\}=h_{4}$.

$S$-functions have long been of great importance in invariant theory [3]. More recently, they have been used in the applications of group theory to physics (e.g. [1], [9]), and in enumeration problems in combinatorics. The combinatorial applications are well reviewed by Read [8]. Most of these applications depend on certain operations defined on $S$-functions, and whether or not they can be readily performed. We will consider two of these operations, the ordinary product and the plethysm (wreath product).

2. Ordinary Product. If we multiply two $S$-functions (this is the ordinary algebraic multiplication of two symmetric functions), the result can be expressed as a linear combination of $S$-functions:

Received August 9, 1971.

AMS (MOS) subject classifications (1970). Primary 20C30; Secondary 20-40.

Key words and phrases. $S$-function, Schur-function, symmetric group, homogeneous productsum, symmetric function, plethysm, wreath product, lattice permutation.

Copyright (c) 1973, American Mathematical Society 


$$
\{\lambda\}\{\mu\}=\sum_{|\nu|} \Gamma_{\lambda \mu \nu}\{\nu\}
$$

There is a convenient algorithm for the coefficients $\Gamma_{\lambda \mu \nu}$ in this ordinary product. An Algol implementation of this rule has been described [2]; here we give a description of the algorithm and discuss an approach to programming it. The result is essentially the same as that given in [2].

First we define two concepts: the Young diagram, and lattice permutations.

Let $\left(\lambda_{1}, \lambda_{2}, \cdots, \lambda_{n}\right), \lambda_{1} \geqq \lambda_{2} \geqq \lambda_{3} \geqq \cdots \geqq \lambda_{n} \geqq 0$ be a partition of $n$. The Young diagram consists of $n$ rows of some convenient symbol, say ' $\mathrm{x}$ ', the $i$ th row containing $\lambda_{i}$ symbols, the rows being left-justified. Thus the Young diagram of $\{4,3,1\}$ is

$$
\begin{array}{llll}
\mathbf{X} & \mathbf{X} & \mathbf{X} & \mathbf{X} \\
\mathbf{X} & \mathbf{X} & \mathbf{X} & \\
\mathbf{X} & & &
\end{array}
$$

The diagram is regular if the length of successive rows is nonincreasing.

A lattice permutation of the $\mu_{1}$ symbols $\alpha, \mu_{2}$ symbols $\beta, \mu_{3}$ symbols $\gamma, \cdots$ is a permutation such that at any stage the number of $\alpha$ 's $\geqq$ number of $\beta$ 's $\geqq$ number of $\gamma$ 's, etc.

For example (reading left to right), $\alpha \alpha \beta \gamma \alpha \beta$ is a lattice permutation of $\alpha^{3} \beta^{2} \gamma$, while $\alpha \beta \gamma \beta \alpha \alpha$ is not, since at the fourth stage we have $\alpha \beta \gamma \beta$ in which the number of $\beta$ 's is 2 , while the number of $\alpha$ 's is 1 .

THEOREM 1 (LiTTLEWOOD-RICHARDSON). The S-functions of $\left\{\lambda_{1}, \lambda_{2}, \cdots, \lambda_{m}\right\}$ $\left\{\mu_{1}, \mu_{2}, \cdots, \mu_{n}\right\}$ are those which correspond to the Young tableaux that can be built by adding to a Young tableau corresponding to $\{\lambda\}, \mu_{1}$ identical symbols $\alpha, \mu_{2}$ identical symbols $\beta, \mu_{3}$ identical symbols $\gamma$, etc., subject to two conditions:

Firstly, after the addition of each set of identical symbols, we must have a regular Young tableau with no two identical symbols in the same column.

\begin{tabular}{|c|c|c|c|c|c|c|c|c|c|c|c|c|c|c|c|c|c|c|c|c|}
\hline $\mathbf{x}$ & $\mathbf{x}$ & $\mathbf{X}$ & $\alpha$ & $\alpha$ & $\mathbf{x}$ & $\mathbf{X}$ & $\mathrm{X}$ & $\alpha$ & $\alpha$ & $\mathbf{X}$ & $\mathbf{X}$ & $\mathbf{X}$ & $\alpha$ & $\mathbf{X}$ & $\mathbf{x}$ & $\mathbf{x}$ & $\alpha$ & $\mathbf{x}$ & $\mathbf{x}$ & $\mathbf{x}$ \\
\hline $\mathbf{x}$ & $\mathbf{x}$ & $\beta$ & & & $\mathbf{x}$ & $\mathbf{x}$ & & & & $\mathrm{x}$ & $\mathbf{x}$ & $\alpha$ & $\beta$ & $\mathbf{x}$ & $\mathrm{x}$ & $\alpha$ & & $\mathbf{x}$ & $\mathbf{x}$ & $\beta$ \\
\hline & & & & & $\beta$ & & & & & & & & & $\beta$ & & & & $\alpha$ & & \\
\hline $\mathbf{X}$ & $\mathbf{x}$ & $\mathbf{X}$ & $\alpha$ & & $\mathbf{x}$ & $\mathbf{x}$ & $\mathbf{x}$ & $\alpha$ & & $\mathbf{X}$ & $\mathbf{x}$ & $\mathbf{X}$ & & $\mathbf{x}$ & $\mathbf{x}$ & $\mathbf{X}$ & & $\mathbf{X}$ & $\mathbf{x}$ & $\mathbf{X}$ \\
\hline $\mathbf{X}$ & $\mathbf{X}$ & & & & $\mathbf{x}$ & $\mathbf{X}$ & & & & $\mathbf{x}$ & $\mathrm{X}$ & $\alpha$ & & $\mathbf{x}$ & $\mathrm{x}$ & $\alpha$ & & $\mathbf{X}$ & $\mathbf{x}$ & \\
\hline$\alpha$ & $\beta$ & & & & $\alpha$ & & & & & $\alpha$ & $\beta$ & & & $\alpha$ & & & & $\alpha$ & $\alpha$ & \\
\hline & & & & & $\beta$ & & & & & & & & & $\beta$ & & & & $\beta$ & & \\
\hline
\end{tabular}

Secondly, if the total set of added symbols is read from right to left in the consecutive rows of the final tableau, we must obtain a lattice permutation of $\alpha^{\mu_{1}} \beta^{\mu_{2}} \gamma^{\mu_{3}} \cdots$.

Thus, for the product $\{3,2\}\{2,1\}$, we obtain

so that

$$
\begin{aligned}
\{3,2\}\{2,1\}=\{5,3\} & +\{5,2,1\}+\{4,4\}+2\{4,3,1\} \\
& +\{4,2,2\}+\{4,2,1,1\} \\
& +\{3,3,2\}+\{3,3,1,1\} \\
& +\{3,2,2,1\} .
\end{aligned}
$$


We shall describe a procedure for the product $\{\lambda\}\{\mu\}$, where $\{\lambda\} \equiv\left\{\lambda_{1}, \lambda_{2}, \cdots, \lambda_{m}\right\}$, $\{\mu\}=\left\{\mu_{1}, \mu_{2}, \cdots, \mu_{n}\right\}$, and use the product calculated above as an example.

We work with partitions (so that the $S$-function $\{3,2\}$ is represented by the numbers 3,2 ), and control regularity by calculating associated difference numbers, defined for the partition $\left(\lambda_{1}, \lambda_{2}, \cdots, \lambda_{n}\right)$ by $d_{i}=\lambda_{i}-\lambda_{i+1}(i=1,2, \cdots, n-1), d_{n}=\lambda_{n}$.

In essence, the method is to apply the first symbol ( $\alpha$ in the example) to the Young diagram of $\{\lambda\}$. This gives a number of functions, to each of which we apply the second symbol, and obtain, in a cascading process, a string of $S$-functions. We continue in this way until there are no more symbols.

Note that the maximum possible length for an $S$-function of the product is $m+n$. We therefore begin by 'stretching' the $S$-function, i.e., adding zeroes to the partition representing $\{\lambda\}$ to give $\left(\lambda_{1}, \lambda_{2}, \cdots, \lambda_{m}, 0,0, \cdots, 0\right)$.

Difference numbers are calculated for each added symbol, so that it is necessary to reference the difference numbers by two subscripts. We shall call the $j$ th difference number for the $i$ th added symbol, $\boldsymbol{d}_{i j}$.

In our example, $(3,2)$ is first 'stretched' to $(3,2,0,0)$ and the first difference numbers are

$$
d_{11}=1, \quad d_{12}=2, \quad d_{13}=0, \quad d_{14}=0 .
$$

Addition of the first symbol will give us a number of products. We generate the first of these as $\left(\lambda_{1}+\mu_{1}, \lambda_{2}, \cdots, \lambda_{m}, 0,0, \cdots, 0\right)$. In our example, this is $(5,2,0,0)$. The procedure for obtaining other partitions is simple and we will confine ourselves to an example.

We repeatedly subtract 1 from the first number and add 1 to the second, provided that the result of the subtraction is not less than the original $\lambda$, i.e., 3 , and that the total added to the second number does not exceed $d_{11}$.

In this way we obtain from $(5,2,0,0):(4,3,0,0)$.

We now use these partitions for the next stage: Subtract 1 from the first element and add it to the third, continuing as above until we arrive at the original 3 or until the difference number $d_{12}$ is exceeded. We obtain

$$
\begin{aligned}
& \text { from }(5,2,0,0):(4,2,1,0),(3,2,2,0) \\
& \text { from }(4,3,0,0):(3,3,1,0) .
\end{aligned}
$$

This completes addition of the first symbol since $d_{13}=0$.

When we come to the addition of subsequent symbols, however, we have to recall the restriction to lattice permutations. We deal with this problem in the following way: For the addition of the $i$ th symbol $(i>1)$ let $k_{r i}$ be the number of $(i-1)$ th symbols added to the $r$ th row, with $k_{r 0}=\lambda_{r}$, and set

$$
c_{p+1}=\sum_{r=1}^{p} k_{r i}, \quad c_{1}=0 .
$$

Put $\bar{c}_{p}=\sum_{i=1}^{n}\left(k_{p i}-k_{p+1, i}\right), \bar{c}_{0}=0$, and let $d_{i j}=\min \left(c_{i}, \bar{c}_{i-1}\right)$.

This modified difference vector will now ensure that both the regularity and the lattice conditions are observed.

For example, in our product $\{3,2\}\{2,1\}$, addition for the first symbol $\alpha$ gives the following tableaux: 


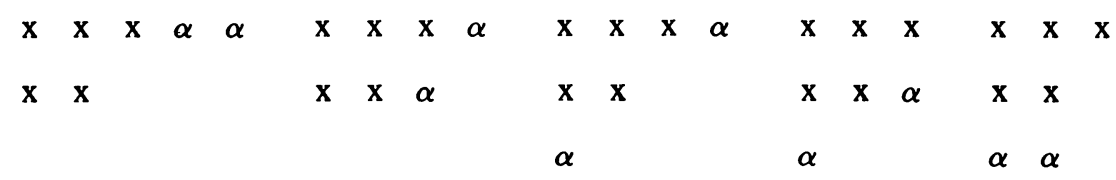

For the first tableau $k_{10}=3, k_{20}=2, k_{30}=0, k_{40}=0, k_{11}=2, k_{21}=0, k_{31}=0$, $k_{41}=0$.

Thus $c_{1}=0, c_{2}=2, c_{3}=2, c_{4}=2, \bar{c}_{1}=3, \bar{c}_{2}=2, \bar{c}_{3}=0, \bar{c}_{4}=0$ so that $d_{21}=0$, $d_{22}=2, d_{23}=2$.

Using these differences numbers, we add $\beta$ 's to the first tableau, thereby creating a number of new tableaux. We then move on to the second tableau,

$$
\begin{array}{llll}
\mathrm{X} & \mathrm{X} & \mathrm{X} & \boldsymbol{\alpha} \\
\mathrm{X} & \mathrm{X} & \boldsymbol{\alpha} &
\end{array}
$$

and repeat the process.

Some collating has to be done: An $S$-function such as $\{4,3,1\}$ is produced in two ways, once as

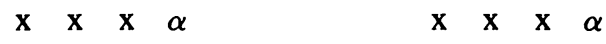

$$
\begin{aligned}
& \begin{array}{lllllllllll} 
& x & \beta & \text { and once as } & \mathrm{x} & \mathrm{x} & \alpha
\end{array} \\
& \alpha \\
& \beta
\end{aligned}
$$

For this reason, an inversion of the process is to be recommended. One can produce partitions in lexicographic order (i.e., in order as numbers, so that for example $(11,2)$ precedes $(10,3)$ since $112>103)$ ([5], [6]). Now the $S$-functions occuring in $\{\lambda\}\{\mu\}$, where $(\lambda)$ is a partition of $n$ and $(\mu)$ a partition of $m$, correspond to partitions of $m+n$. Thus one produces one such partition at a time, and using a slight variation of the above procedure, finds how many times it occurs in the product. Then on to the next partition, and so on, until there are no more partitions. At the end, we have a list of partitions in lexicographic order together with their coefficient $\Gamma_{\lambda \mu \nu}$.

3. The Plethysm (Wreath Product). The plethysm was first defined by Littlewood in the context of invariant matrices (see [3], [4]) and representations of the full linear group. An equivalent definition [8] is of the wreath product, defined for any two polynomials $A$ and $B$ in indeterminates $s_{1}, s_{2}, \cdots, s_{n}: A[B]$ is the polynomial we get by replacing every $s_{r}$ in $A(r=1,2, \cdots)$ by the polynomial obtained from $B$ by multiplying the subscript of each of its indeterminates by $r$. Thus if

$$
A=\frac{1}{2}\left(s_{1}^{2}+s_{2}\right), \quad B=\frac{1}{3}\left(s_{1}^{3}+2 s_{3}\right)
$$

the 'substitution' is effected by replacing

$$
s_{1} \text { by } \frac{1}{3}\left(s_{1}^{3}+2 s_{3}\right) \text { and } s_{2} \text { by } \frac{1}{3}\left(s_{2}^{3}+2 s_{6}\right)
$$

in $A$. The result is

$$
\frac{1}{2}\left\{\frac{1}{3}\left(s_{1}^{3}+2 s_{3}\right)^{2}+\frac{1}{3}\left(s_{2}^{2}+2 s_{6}\right)\right\} .
$$

Now every $S$-function, being a symmetric function, can be expressed as a polynomial in the power-sum symmetric function $s_{r}$. In this way we define the wreath 
product $\{\lambda\}[\{\mu\}]$ (in plethysm notation, $\{\mu\} \otimes\{\lambda\}$ ) of $\{\lambda\}$ and $\{\mu\}$.

The result is reconverted into a sum of $S$-functions: $\{\lambda\}[\{\mu\}]=\sum_{(\nu)} c_{\lambda \mu \nu}\{\nu\}$, where the $c_{\lambda \mu \nu}$ are numerical coefficients.

It can be shown, if $\{\lambda\}$ is a partition of $n$ and $\{\mu\}$ a partition of $m$, that $c_{\lambda_{\mu \nu}}=0$ unless $\{\nu\}$ is a partition of $m n$. Note that the product is noncommutative. We have, however,

$$
(\{\lambda\}+\{\mu\})[\{\nu\}]=\{\lambda\}[\{\nu\}]+\{\mu\}[\{\nu\}]
$$

and

$$
\{\lambda\}\{\mu\}[\{\nu\}]=\{\lambda\}[\{\nu\}]\{\mu\}[\{\nu\}] .
$$

The problem of determining the coefficients $c_{\lambda \mu \nu}$ is difficult. In many special cases there are a number of useful general theorems, a survey of which will be found in [6]. In other special cases, methods well suited to a computer have been derived ([6], [7]). We shall follow here a general method discussed in [8], and will confine ourselves to wreath products of one-part $S$-functions. For these we will use the notation $h_{m}\left[h_{n}\right]\left(\right.$ since $\left.h_{m}=\{m\}\right)$.

4. The Plethysm $s_{m}\left[h_{n}\right]=\{n\} \otimes s_{m}$. As a preliminary, we discuss a theorem of Todd [11] for the special wreath product $s_{m}\left[h_{n}\right]$ where $s_{m}$ denotes the power-sum symmetric function. A special case of Todd's theorem can be reconstrued as follows:

THEOREM 2. Let $\sigma \equiv\left(\sigma_{1}, \sigma_{2}, \cdots, \sigma_{m}\right)$ be a partition of $m n$ into at most $m$ parts. Let $\left(n_{1}, n_{2}, \cdots, n_{m}\right)$ be a partition of $n$ into at most $m$ parts such that $r_{i}=$ $\sigma_{i}-m\left(n_{i}-1\right)-i$ satisfies $0 \leqq r_{i} \leqq m-1$. Let $\theta_{\sigma}$ be the sign of the permutation

$$
\left(\begin{array}{c}
r_{1}, r_{2}, \cdots, r_{n} \\
m-1, m-2, \cdots, 0
\end{array}\right)
$$

(or zero if the $r_{i}$ are not a permutation of $(m-1, m-2, \cdots, 0)$ ). Then $s_{m}\left[h_{n}\right]=$ $\sum \theta_{\sigma}\{\sigma\}$.

To program this efficiently we produce, one at a time, partitions $(\sigma)$ of $m n$ into $m$ parts. We need also the partition $\left(n_{1}, n_{2}, \cdots, n_{m}\right)$ of $n$. We can show that, if $n_{i}$ exists, then $n_{i}=\left[\left(\sigma_{i}+m-i\right) / m\right]$ where brackets denote "integer part." For if we put

$$
r_{i}=m \frac{\sigma_{i}+m-i}{m}-m\left[\frac{\sigma_{i}+m-i}{m}\right] \geqq 0, \text { then } \quad r_{i}<m .
$$

If the $n_{i}$ so chosen are in fact a partition of $n$ then

$$
\begin{aligned}
\sum r_{i} & =\sum\left(\sigma_{i}-m\left(n_{i}-1\right)-i\right) \\
& =n m-m n+m^{2}-\frac{1}{2} m(m+1) \\
& =0+1+2+\cdots+(m-1) .
\end{aligned}
$$

If not, then at least two of the $r_{i}$ must be equal. Hence we calculate $c_{i j}=r_{i}-r_{i}$ $(i=1,2, \cdots, m-1 ; j>i)$. Let $k$ be the number of values for which $c_{i j}<0$. Then

$$
\begin{aligned}
\theta_{\sigma} & =(-1)^{k} & & \text { if no } c_{i i}=0, \\
& =0 & & \text { otherwise. }
\end{aligned}
$$


Thus, provided we have a program to produce partitions, calculating the expansion is not difficult.

Todd (ibid.) also showed that there exists a 1-1 correspondence between those $S$-functions $\left\{\sigma_{1}, \sigma_{2}, \cdots, \sigma_{m}\right\}$ of $\{n\} \otimes s_{m}$ for which $\sigma_{m}>0$ and the $S$-functions of $\{n-1\} \otimes s_{m}$. For the $S$-functions of $\{n\} \otimes s_{m}$ for which $\sigma_{m}=0$, there exists a 1-1 correspondence with the $S$-functions of $\{n\} \otimes s_{m-1}$. From this we derive the following interesting theorem:

THEOREM 3. Let $\{n\} \otimes s_{m}=\sum_{\sigma} \theta_{\sigma}\{\sigma\}$. Let $u(n, m)$ be the number of nonzero $\theta_{\sigma}$. Then

$$
u(n, m)=\left(\begin{array}{c}
n+m-1 \\
n
\end{array}\right)
$$

Proof. Todd's result implies

$$
u(n, m)=u(n-1, m)+u(n, m-1) .
$$

This remains true if as usual we define $u(n, m)=0$ if $n<0$ or $m<0 ; u(n, 0)=1$ if $n>0$. However, we define $u(0, m)=0$ for all $m$.

Also, since $\{n\} \otimes s_{1}=\{n\}, u(n, 1)=1$. Write

$$
\begin{aligned}
& P_{m}(x)=\sum_{n=0}^{\infty} u(n, m) x^{n}, \\
& P_{m}(x)=P_{m-1}(x)+x P_{m}(x), \\
& P_{m}(x)=P_{m-1}(x) /(1-x) .
\end{aligned}
$$

Now

$$
P_{1}(x)=\sum_{n=0}^{\infty} u(n, 1) x^{n}=\sum_{n=0}^{\infty} x^{n}=1 /(1-x)
$$

Therefore

$$
P_{m}(x)=\frac{1}{(1-x)^{m}}=\sum_{r=0}^{\infty}\left(\begin{array}{c}
m+r-1 \\
r
\end{array}\right) x^{r}
$$

so that $u(n, m)$, the coefficient of $x^{n}$ in $P_{m}(x)$, is $\left({ }_{n}^{n+m-1}\right)$ which proves the theorem.

This result is very useful in checking expansions. Use of a similar procedure gives the number of functions in $\{n\} \otimes s_{m}$ with a given sign.

5. The Plethysm $h_{m}\left[h_{n}\right]$. Now that we have the functions $s_{m}\left[h_{n}\right]$, it is possible to obtain recursively the general plethysm $h_{m}\left[h_{n}\right]$. A well-known relation [3] is

$$
m h_{m}=\sum_{r=1}^{m} s_{r} h_{m-r}
$$

Combining this with the distributive properties of the plethysm [4], we get

$$
m h_{m}\left[h_{n}\right]=\sum_{r=1}^{m} s_{r}\left[h_{n}\right] h_{m-r}\left[h_{n}\right]
$$

Assume that we have calculated and stored the functions $s_{r}\left[h_{n}\right]$ for $r=1,2, \cdots, m$. To begin, we know $h_{0}\left[h_{n}\right]=\{0\}, h_{1}\left[h_{n}\right]=\{n\}$. For example, since $s_{1}\left[h_{2}\right]=\{2\}$, 
$s_{2}\left[h_{2}\right]=\{4\}-\{3,1\}+\{2,2\}$ and $h_{1}\left[h_{2}\right]=\{2\}$, we have $2 h_{2}\left[h_{2}\right]=(\{2\}\{2\})$ $+(\{4\}-\{3,1\}+\{2,2\})(\{0\})$. The full expansion is now obtained by multiplying the relevant $S$-functions.

In practice, as explained at the end of Section 3, it is better to take each partition of $m n$ in turn and find its total coefficient. Thus, in the above example, we would begin with $\{4\}$, find that it occurs once in $\{2\}\{2\}$, once in $\{4\}\{0\}$ and not elsewhere, so that the coefficient of $\{4\}$ in $h_{2}\left[h_{2}\right]$ is 1 .

The advantages of this variation are even more marked by noting that it is easy to tell when a function does not occur in a given product: if it is too long, too 'broad', too 'short' or too 'thin'. In $\{2,2\}\{0\}$ the function $\{4\}$ cannot occur since it is too 'short'; it is of length 1 whereas each product should be of length 2.

In either approach, some preliminary sorting is advisable to avoid duplication of multiplication. Thus in obtaining the expansion $h_{3}\left[h_{3}\right]$, since

$$
\begin{array}{ll}
s_{2}\left[h_{3}\right]=\{6\}+\{5,1\}+\{4,2\}+\{3,3\}, & s_{1}\left[h_{3}\right]=\{3\}, \\
h_{2}\left[h_{3}\right]=\{6\}+\{4,2\}, & h_{1}\left[h_{3}\right]=\{3\},
\end{array}
$$

$\{6\}\{3\}$ occurs in both $s_{2}\left[h_{3}\right] h_{1}\left[h_{3}\right]$ and $s_{1}\left[h_{3}\right] h_{2}\left[h_{3}\right]$.

6. Conclusion. The procedures outlined above have all been programmed and tables of the plethysm obtained for $h_{m}\left[h_{n}\right]$ in the following cases: $n=4$ or 5 , $m \leqq 6 ; n=6,7$ or $8, m \leqq 5$. Different methods ([6], [7]) were used to produce $h_{m}\left[h_{3}\right.$ ], $m \leqq 10$. These have all been included as a large appendix to [6].

Acknowledgment. The author wishes to thank Professor R. C. Read for his advice and encouragement.

Department of Mathematics

University of the West Indies

St. Augustine, Trinidad, West Indies

1. P. H. BUtLER, "S-functions and symmetry in physics," J. Physique (Supp. to Nos. 11-12) Nov.-Dec. 1970, C4-47 to 49.

2. D. B. HUNTER, "Analysis of the outer product of symmetric group representations," Nordisk. Tidskr. Informationsbehandling, v. 10, 1970, pp. 106-114.

3. D. E. LiTtLEwOod, A University Algebra, Heinemann, Melbourne, 1950. MR 13, 523. 1950.

4. D. E. LitrLewOoD, Theory of Group Characters, 2nd ed., Clarendon Press, Oxford, 1970.

5. J. K. S. MCKay, "Partitions in natural order (Algorithm 371)," Comm. ACM, v. 13,

6. P. A. MoRrIS, Graph Theoretic and Computer Applications to S-function Theory, Ph.D. Thesis, University of the West Indies, 1970.

7. P. A. MoRrIs, "Applications of graph" theory of $S$-function theory, J. London Math. Soc. (To appear.)

8. R. C. READ, "The use of $S$-functions in combinatorial analysis," Canad. J. Math., v. 20, 1968, pp. 808-841, MR 37 \#5108.

9. P. R. SMith \& B. G. WyBouRne, "Plethysm and the theory of complex spectra," J. Mathematical Phys., v. 9, 1968, pp. 1040-1051.

10. P. R. SMITH \& B. G. WYBOURNE, "Selection rules and decomposition of the Kronecker square of irreducible representations," J. Mathematical Phys., v. 8, 1967, pp. 2434-2440.

11. J. A. TODD, "A note on the algebra of $S$-functions," Proc. Cambridge Philos. Soc., v. 45,1949 , pp. 328-334. MR 10, 672. 